\title{
Treatment with Enriched Environment Reduces Neuronal Apoptosis in the Periinfarct Cortex after Cerebral Ischemia/ Reperfusion Injury
}

\author{
Xiuping Chen ${ }^{\mathrm{a}}$ Xin Zhang ${ }^{\mathrm{a}}$ Li Xue $^{\mathrm{b}}$ Chizi Hao ${ }^{\mathrm{a}}$ Weijing Liao ${ }^{\mathrm{a}}$ Qi Wanc
}

aDepartment of Rehabilitation Medicine, Zhongnan Hospital of Wuhan University, ${ }^{\mathrm{b}}$ Department of Rehabilitation Medicine, Puai Hospital, Tongji Medical College, Huazhong University of Science and Technology, 'Department of Physiology, Collaborative Innovation Center for Brain Science, School of Basic Medical Sciences, School of Medicine, Wuhan University, Wuhan, Hubei, China

\section{Key Words}

Enriched environment - Cerebral ischemia - Neuronal apoptosis - Middle cerebral artery occlusion - Mitochondria

\begin{abstract}
Background/Aims: Enriched environment (EE) has been reported to exert neuroprotective effect in animal models of ischemic stroke. However, the underlying mechanism remains unclear. The purpose of this study was to investigate the effect of EE treatment on neuronal apoptosis in the periinfarct cortex after cerebral ischemia/reperfusion (I/R) injury. Methods: The cerebral I/R injury was established by middle cerebral artery occlusion (MCAO). A set of behavioral tests including the modified neurological severity score (mNSS), limb-placing test and foot-fault test were conducted. The infarct volume and the neuronal survival rate were evaluated by Nissl staining. The morphology and ultrastructure of ischemic neurons was examined by transmission electron microscopy. Neuronal apoptosis was assessed by double labeling of TdT-mediated dUTP-biotin nick end labeling (TUNEL) with NeuN. The expressions of apoptosis-related proteins were tested by western blotting and immunohistochemical labeling. Results: EE treatment improved neurological function, reduced infarct volume, increased neuronal survival rate and alleviated the morphological and ultrastructural damage of neurons (especially mitochondria) after $\mathrm{I} / \mathrm{R}$ injury. EE treatment reduced the neuronal apoptosis, increased $\mathrm{B}$ cell lymphoma/leukemia-2 (BCl-2) protein levels while decreased $\mathrm{BCl}-$ 2 -associated $\mathrm{X}$ protein (Bax), cytochrome $\mathrm{c}$, caspase-3 expressions and $\mathrm{Bax} / \mathrm{Bcl}-2$ ratio in the periinfarct cortex after cerebral I/R injury. Conclusion: Our findings suggest that EE treatment inhibits neuronal apoptosis in the periinfarct cortex after focal cerebral I/R injury, which may be one of the possible mechanisms underlying the neuroprotective effects of EE.




\section{Cellular Physiology Cell Physiol Biochem 2017;41:1445-1456 \\ and Biochemistry Published \begin{tabular}{l|l} 
DOI: 10.1159/000468368 & $\begin{array}{l}\text { C } 2017 \text { The Author(s). Published by S. Karger AG, Basel } \\
\text { www.karger.com/cpb }\end{array}$
\end{tabular} \\ Chen et al.: Enriched Environment Reduces Neuronal Apoptosis in Rats}

\section{Introduction}

Cerebral ischemic stroke is a frequently occurring disease characterized by high mortality, morbidity, disability and reoccurrence [1]. Although significant advances in the technological and medical treatments have been made, many patients still suffer from varying degrees of dysfunction, which directly weaken their quality of life [2]. Therefore, it is extremely important to find effective strategies to improve the functional recovery of stroke patients.

Apoptosis is a form of programmed cell death characterized by cytoplasmic blebbing, chromatin condensation and DNA fragmentation [3]. It is essential in normal tissue development and homeostasis, while inappropriate or excessive apoptosis accelerates cell death [4]. After cerebral ischemia, the outer membrane of mitochondria becomes permeabilized, resulting in the release of cytochrome $c$ from mitochondria intermembrane space to the cytoplasm, which subsequently activates caspase-3, and finally leads to DNA fragmentation [5]. This process of protein translocation and activation is mediated by the Bcl-2 family members such as antiapoptotic protein B cell lymphoma/leukemia-2 (Bcl-2) and proapoptotic protein Bcl-2-associated X protein (Bax) [6]. Human studies have suggested that patients with less apoptosis-positive cells in the penumbra were coupled with prolonged survival time [7]. Therefore, inhibition of apoptosis may be a promising strategy to promote poststroke recovery.

An enriched environment (EE) is an effective rehabilitative protocol for rodents, in which multiple animals are housed together in a large space equipped with different toys and receive more sensorimotor, cognitive and social stimulation compared with the standard conditions [8]. Mounting studies have suggested that exposure to EE treatment after brain injury leads to neuroprotective effects in animal models $[9,10]$. Recently, EE treatment has been translated into a rehabilitation strategy for poststroke patients in the clinical setting [11]. The underlying mechanisms of EE treatment-induced neuroprotection after cerebral ischemia are multifaceted. Our previous study has demonstrated that EE treatmentinduced angiogenesis contributed to the improved functional recovery of stoke animals [12]. However, whether the neuroprotective effect of EE treatment is associated with the reduction of neuronal apoptosis in the periinfarct cortex has not been reported.

In the present study, we investigated the effect of EE treatment on neuronal apoptosis in the periinfarct cortex after cerebral ischemia/reperfusion (I/R) injury. We examined neurological deficits and infarct volume. We performed transmission electron microscope to determine the morphology and ultrastructure of neurons in the periinfarct area of cortex. We tested neuronal apoptosis in the periinfarct cortex using double immunofluorescent staining of TUNEL with NeuN. The immunostaining and western blot were also employed to determine the protein levels of Bcl-2, Bax, cytochrome $c$ and caspase-3 in the periinfarct cortex at 14 days after cerebral I/R injury.

\section{Materials and Methods}

\section{Animals}

Male Sprague-Dawley (SD) rats weighing 200-240g were purchased from Hunan SJA Laboratory Animal Co., Ltd (SCXK (Xiang 2013-0004)). All rats were housed in a controlled condition (4 animals per cage, $22-25^{\circ} \mathrm{C}, 50-60 \%$ relative humidity, $12 \mathrm{~h}$ light/dark cycle) with free access to water and standard rat chow for 3 days to adapt to the laboratory environment before receiving operation. All experimental procedures were performed under the Guidance Suggestion for the Care and Use of Laboratory Animals. The experimental protocol for this research was approved by the Institutional Animal Care and Use Committee of Wuhan University, China. All efforts were made to reduce the mortality of animals and their suffering.

Groupings and housing conditions

Animals were randomly divided into 3 groups: sham group (sham-operated rats housed in standard condition), I/R group (middle cerebral artery occlusion (MCAO) rats housed in standard condition), or EE 


\section{Cellular Physiology Cell Physiol Biochem 2017;41:1445-1456 \\ and Biochemistry Publisnec onl004ne:TVarch 17, $2017, \begin{aligned} & \text { DO 2017 The Author(s). Published by S. Karger AG, Basel } \\ & \text { www.karger.com/cpb }\end{aligned}$ \\ Chen et al.: Enriched Environment Reduces Neuronal Apoptosis in Rats}

group (MCAO rats housed in EE). Twenty-four hours after MCAO, rats in EE group went to EE cages, which were $90 \mathrm{~cm}$ long $\times 75 \mathrm{~cm}$ wide $\times 50 \mathrm{~cm}$ high with wire mesh lids containing climbing ladders, chains, different shaped tubes, plastic tunnels, colored blocks and a running wheel to provide sensorimotor stimulations. EE also provided enhanced social stimulation by housing 6-8 rats together in one cage. The contents were changed daily to promote novelty and exploration. Rats in I/R and sham groups were housed in standard cages $(44 \mathrm{~cm}$ long $\times 32 \mathrm{~cm}$ wide $\times 20 \mathrm{~cm}$ high) with no objects, 4 rats per cage.

\section{MCAO model}

All rats received right MCAO as previously described [13]. A total of 60 healthy adult male SD rats were anesthetized using isoflurane administered through a face mask. A midline incision about $2 \mathrm{~cm}$ was made in the neck skin, and the right carotid artery (CCA), external carotid artery (ECA), and internal carotid artery (ICA) were carefully isolated. After that, a monofilament nylon filament (Beijing Cinontech Biotech Co., Ltd., Beijing, China) was inserted through the right CCA and gently advanced into the ICA to a point about $18 \mathrm{~mm}$ distal to the bifurcation of the carotid artery, the right MCA was then occluded. $2 \mathrm{~h}$ later, the filament was gently withdrawn for reperfusion. Rats in sham group received the same surgical procedures except inserting a nylon filament. During the surgery, seven rats (three in sham group, two in I/R group and two in EE group) died of excessive bleeding and vagus nerve cut off. After recovering from anesthesia, the neurologic results were scored on a five-point scale and the rats were excluded from the study with a score of 0 or 4 [14]. Rats with scores of 1-3 were considered successful models and included in the study. Five rats (two in I/R group and three in EE group) with scores of 0 or 4 were excluded. During the following two weeks, six rats (three in sham group, two in I/R group and one in EE group) died at the first three days and no more death after that. Finally, forty-two rats were included in this study, and the number of rats in each group was fourteen.

\section{Behavioral tests}

The mNSS was performed at 3, 7, 14 days after stroke by an investigator who was blinded to the experimental groups [15]. It is a composite of motor, sensory, reflex and balance tests. The total score is 18, which indicates the most severe injury. One score point is scored for failure to perform the test or the lack of a tested reflex. Thus, a higher score indicates a more severe injury.

Foot-fault test was utilized as a measure of motor impairment which was widely used after stroke [16]. The test consists of free movement on a grid-like surface for $5 \mathrm{~min}$. When a rat places the limbs incorrectly on the grid, the limb falls through and is counted as a fault. The test was conducted post-operative days 3,7 , and 14 . Tests were always performed twice and means were used for statistical analysis.

Limb-placing test was used to assess the sensorimotor integration of forelimb and hindlimb responses to tactile and proprioceptive stimulation, which consists of seven limb-placing tasks [17]. The tasks were scored as follows: 2 points: the rat performed normally; 1 point, the rat performed with a delay ( $2 \mathrm{~s}$ ) and/or incompletely; 0 points, the rat did not perform normally. Both sides of the body were included. All rats were pre-trained on these tests for three days before ischemia induction.

\section{Nissl staining}

Rats ( $n=6$ per group) were deeply anesthetized using an intraperitoneal injection of chloral hydrate at 14 days after MCAO and then transcardially perfused with $200 \mathrm{~mL}$ of $0.9 \%$ saline followed by $200 \mathrm{~mL}$ of $4 \%$ paraformaldehyde solution. The brain tissues were harvested and postfixed in paraformaldehyde overnight. Thereafter, $20-\mu \mathrm{m}$ thick coronal sections with the distance between adjacent sections of $400 \mu \mathrm{m}$ were collected for Nissl staining.

The procedure was carried out as previously described [18]. Brain sections were immersed in $0.1 \%$ cresyl violet for $40 \mathrm{~min}$ at $60^{\circ} \mathrm{C}$ and then rinsed in distilled water for $10 \mathrm{~min}$. Stained sections were fixed by serial dehydration in alcohol and xylene before cover slipping with mounted medium. The infarct region was defined as containing dark pyknotic necrotic cell bodies or reduced staining. To minimize the effect of edema and liquefaction resulting from infarction, the infarct area in each section was calculated as: contralateral hemisphere area - ipsilateral non-infarct area. Infarct volume between two adjacent sections was calculated by the formula: $1 / 3 \times \mathrm{h}(\mathrm{S} 1+\mathrm{S} 2+\sqrt{\mathrm{S} 1 * \mathrm{~S} 2}), \mathrm{S} 1$ and $\mathrm{S} 2$ were the infarct areas of two sections, and $\mathrm{h}$ was the distance between them [19]. The total infarct volume was derived from the sum of all infarct volume between each adjacent section. At last, the percentage of infarct volume was calculated as: total infarct volume/total contralateral hemispheric volume $\times 100 \%$. 


\section{Cellular Physiology Cell Physiol Biochem 2017;41:1445-1456

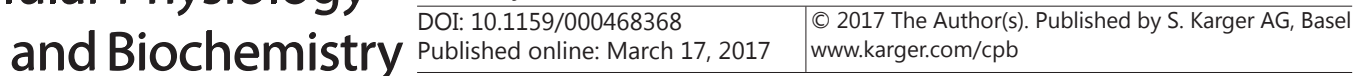 \\ Chen et al.: Enriched Environment Reduces Neuronal Apoptosis in Rats}

For counting the number of viable neurons, four random views in each section were measured per rat using a light microscope (Olympus Corporation, Tokyo, Japan) at $\times 200$ magnification and the number of survival neurons was quantified with Image Pro Plus 6.0 (Media Cybernetics Inc., Bethesda, MD, USA) by an observer without knowledge of the experiment. Only orderly arranged cells had normal morphology with abundant cytoplasm and evident nucleus and nucleolus were considered as survival neurons and included.

\section{Transmission electron microscopy}

Fourteen days after MCAO, fresh brain tissues ( $n=2$ per group) were taken from the periinfarct cortical area, cut into small pieces of $1 \mathrm{~mm} \times 1 \mathrm{~mm} \times 1 \mathrm{~mm}$ and fixed in $2.5 \%$ glutaraldehyde overnight at $4{ }^{\circ} \mathrm{C}$. Then, specimens were rinsed with PBS and soaked in osmium tetroxide. After dehydration in acetone, specimens were embedded in epoxide resin and $70 \mathrm{~nm}$ thick sections were prepared. After that, species were put onto copper grids and stained with uranyl acetate followed by lead citrate. At last, cellular and ultrastructural changes of neurons were observed and photographed by transmission electron microscope (HT7700, Hitachi, Japan).

\section{TUNEL staining}

To evaluate neuronal apoptosis in the periinfarct cortex, double immunofluorescent staining of the TdT-mediated dUTP-biotin nick end labeling (TUNEL) with NeuN was performed. The paraffin sections ( $4 \mu \mathrm{m}$ thick) were incubated overnight at $4^{\circ} \mathrm{C}$ with anti-NeuN antibody (1:150, Abcam, Cambridge, UK), followed by incubation with Cy3-conjugated goat anti-mouse IgG secondary antibodies (1:50, Aspen Biotechnology, Hubei, China) for $50 \mathrm{~min}$ at room temperature. TUNEL staining was performed using an in situ cell death detection kit (Roche, Basel, Switzerland) following the manufacturer's protocol [20]. At last, the sections were covered with 4'6-diamidino-2-phenylindole (DAPI) (Aspen Biotechnology, Hubei, China). In this study, four random fields in the penumbra of ischemic cortex were captured in each section with a fluorescence microscope (Olympus Corporation, Tokyo, Japan). The number of stained cells per field was calculated under higher magnification $(200 \times)$ by an investigator blinded to experiment design.

\section{Western blot}

The experimental rats were euthanized and the periinfarct cortical tissues ( $\mathrm{n}=6$ per group) were removed at 14 days post-MCAO for western blot analysis as previously described [21]. The concentration of proteins was determined by using a protein assay kit (Bio-Rad, USA). $40 \mu \mathrm{g}$ proteins were electrophoresed on SDS-PAGE gels and then transferred onto polyacrylamide difluoride (PVDF) membrane (Millipore, Massachusetts, USA). Membranes were incubated with respective primary antibodies (anti-Bax, 1:1500, CST; anti-Bcl-2, 1:1000, Abcam; anti-cytochrome $c$, 1:1500, CST; anti-caspase-3, 1:1000, Abcam) overnight at $4^{\circ} \mathrm{C}$, followed by incubation with horseradish peroxidase (HRP)-conjugated goat anti-rabbit (1:10000, KPL, USA) or goat anti-mouse IgG secondary antibodies (1:10000, KPL, USA) for $30 \mathrm{~min}$ at room temperature. Protein bands were visualized by an enhanced chemiluminescence system (ECL kit, Aspen Biotechnology, Hubei, China). Quantification of band intensity (optical density) was analyzed using AlphaEaseFC software (Alpha Innotech, California, USA). GAPDH served as loading condition.

\section{Immunohistochemistry and Immunofluorescence}

Brain paraffin sections $(4 \mu \mathrm{m})$ were incubated overnight at $4{ }^{\circ} \mathrm{C}$ with rabbit polyclonal anti-Bcl-2 antibody (1:200, Abcam, Cambridge, UK) and rabbit monoclonal anti-Bax antibody (1:200, Abcam, Cambridge, UK) respectively. After three times rinses (5 min each) with phosphate-buffered saline (PBS, $\mathrm{pH}=7.4)$, the sections reacted with goat anti-rabbit IgG conjugated to peroxidase secondary antibody (1:200, Aspen Biotechnology, Wuhan, China). The remaining procedures were according to the standard procedures [22]. Negative controls were established to investigate the specificity of the reactions. For semi-quantitative analysis, four visual fields $(200 \times)$ from each section in periinfarct area were photographed under a light microscope (Olympus Corporation, Tokyo, Japan), and integrated optical density (IOD) was analyzed using Image Pro Plus 6.0 (Media Cybernetics Inc., Bethesda, MD, USA).

The immunofluorescent staining was used to assess the expression of caspase-3. Brain sections were then incubated overnight with rabbit polyclonal anti-caspase-3 antibody (1:200, Abcam, Cambridge, UK). After being rinsed with PBS, the tissues were incubated with Cy3-conjugated goat anti-rabbit IgG secondary antibody (1:50, Aspen Biotechnology, Wuhan, China). The unspecific labeling was found by replacing the primary antibody with PBS. 
Statistical analysis

All experimental data were analyzed using SPSS20.0 software (SPSS Inc., Chicago, Illinois, USA) and presented as mean \pm standard deviation (SD). Comparisons between groups were analyzed using one-way ANOVA, followed by LSD $t$-test. A student's test was used to analyze differences in infarct volume between the EE and I/R groups. $P<0.05$ was considered to be statistically significant.

\section{Results}

EE treatment improves neurological function in stroke animals

To determine whether EE treatment promoted functional recovery of stroke rats, a series of tests including the mNSS, foot-fault test and limb-placing test were performed. The ischemic rats (EE and I/R) showed worse performance compared to the sham group on all three behavioral tests at various time points after MCAO (Fig. 1A-C). The mNSS scores of sham group at each time point were zero (Fig. 1A). No difference was found between the EE and I/R groups on day 3 (Fig. 1A). However, rats in EE group showed lower mNSS scores than those in I/R group at 7 and 14 days after MCAO (Fig. 1A). The EE group had significant less left forefoot faults (Fig. 1B) and improved performance of limb placement (Fig. 1C) at 7 and 14 days after MCAO compared to the I/R group. Taken together, these findings suggest that EE treatment improves neurological functional recovery of stroke animals.

\section{EE treatment reduces infarct volume and increases neuronal survival \\ As the improved functional outcome} might be ascribed to the reduced brain damage, cerebral infarct volume was evaluated by Nissl staining at 14 days after MCAO (Fig. 2A). The results showed that EE treatment significantly reduced infarct volume compared to I/R group (Fig. 2A). No lesion was found in the sham group.

We further observed the number of viable neurons in the periinfarct cortex. In $I / R$ group, most of the neurons in the periinfarct cortex were shrunk, deeply stained and sparsely distributed with cytosolic concentration, karyopyknosis and karyorrhexis (Fig. 2B), which were seldom observed in EE group. While the number of viable neurons, which were orderly arranged with normal morphology and evident nucleus and nucleolus, was significantly increased in EE group (Fig. 2B). In sham group, normal neurons were orderly arranged with normal morphology and evident nucleus and nucleolus (Fig. 2B). These results suggest that $\mathrm{EE}$ treatment reduces brain damage induced by cerebral I/R injury.

EE treatment protects against cerebral

$I / R$ injury-induced alterations of neuronal morphology and ultrastructure in the periinfarct cortex

In order to determine EE treatmentinduced the stabilization of neuronal

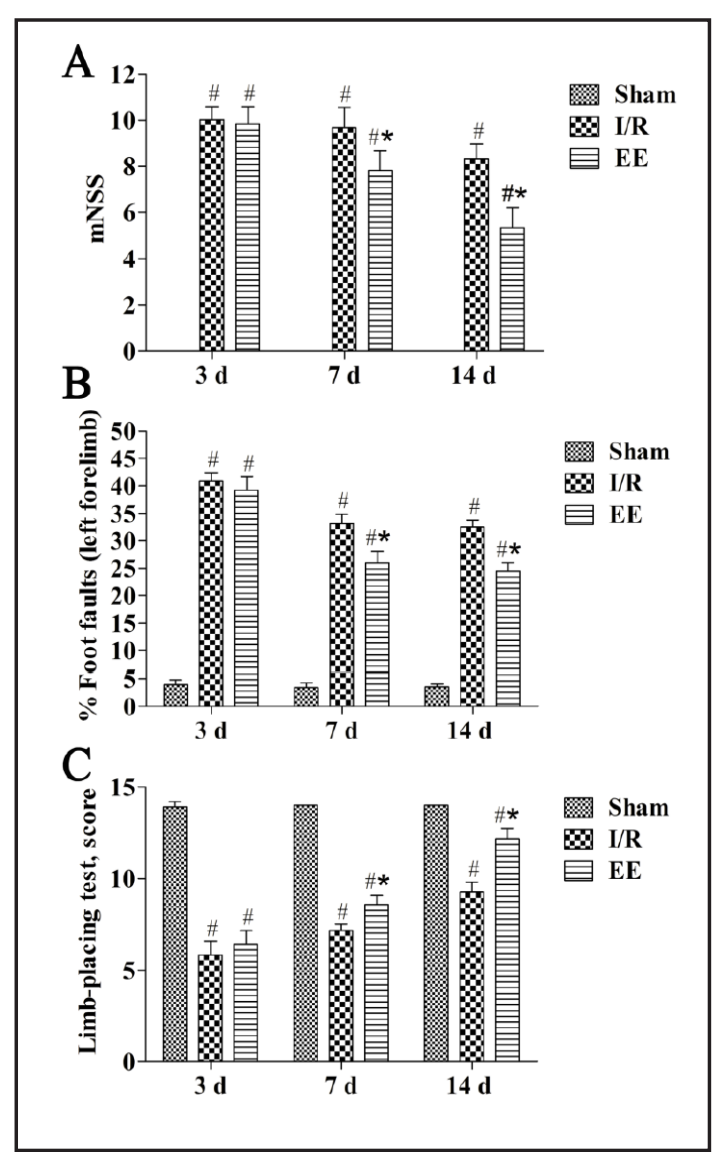

Fig. 1. EE treatment improves neurological deficits at 3,7 and 14 days after MCAO. Quantitative analysis of mNSS (A), foot-fault test (B) and limb-placing test (C). Data were expressed as mean $\pm \mathrm{SD}, \mathrm{n}=14$ /group. ${ }^{\#} P<0.05$ vs sham group, ${ }^{*} P<0.05$ $v s \mathrm{I} / \mathrm{R}$ group. 


\section{Cellular Physiology and Biochemistry}

Cell Physiol Biochem 2017;41:1445-1456

\begin{tabular}{l|l}
\hline DOI: $10.1159 / 000468368$ & C 2017 The Author(s). Published by S. Karger AG, Basel
\end{tabular}

Chen et al: Enriched Environment Reduces Neuronal Apoptosis in Rats

Fig. 2. EE treatment reduces infarct volume and enhances the number of viable neurons at 14 days after MCAO. (A) Representative cresyl violet stained brain slices and quantitative analysis for the percentage of infarct volume in each group. The square ( $\square$ ) indicates the representative area of periinfarct cortex. EE treatment significantly reduced infarct volume compared to I/R group (17.83 \pm $2.04 \%$ vs $28.67 \pm 2.73 \%, P<0.05$ ). (B) Representative photomicrographs were taken at $\times 200$ from the square ( $\square$ ) and quantitative analysis for the number of viable neurons, bar $=50 \mu \mathrm{m}$. Normal neurons were orderly arranged with normal morphology and evident nucleus and nucleolus. Abnormal neurons were shrunk and deeply stained. EE treatment significantly increased the number of viable neurons compared with I/R group (157.17 $\pm 8.23 / \mathrm{mm}^{2}$ vs $76.83 \pm 7.41 / \mathrm{mm}^{2}$, $P<0.05)$. Data were presented as mean \pm SD, $\mathrm{n}=6$ /group. ${ }^{\#} P<0.05$ vs sham group, ${ }^{*} P<0.05$ vs I/R group.

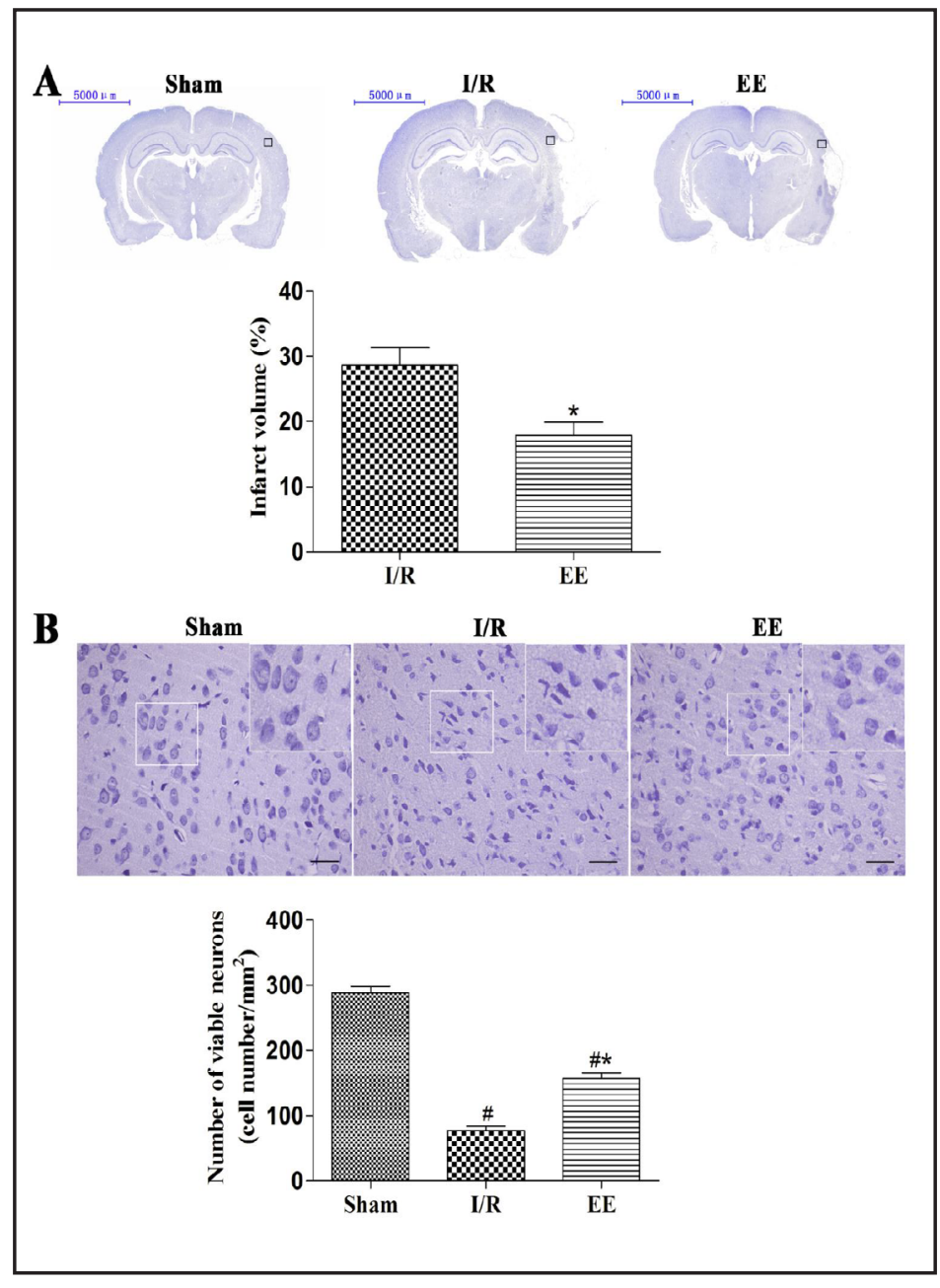

Fig. 3. EE treatment protects morphological and ultrastructural changes of neurons in the periinfarct cortex. Representative photographs of nucleolus and mitochondria of neuron in sham group (A, D), I/R group $(B, E)$ and EE group (C, F) at 14 days after cerebral I/R injury. $\mathrm{n}=2$ per group. Nm: Nuclear membrane; Ch: Chromatin; Mi: Mitochondria; Vc: Vacuole.

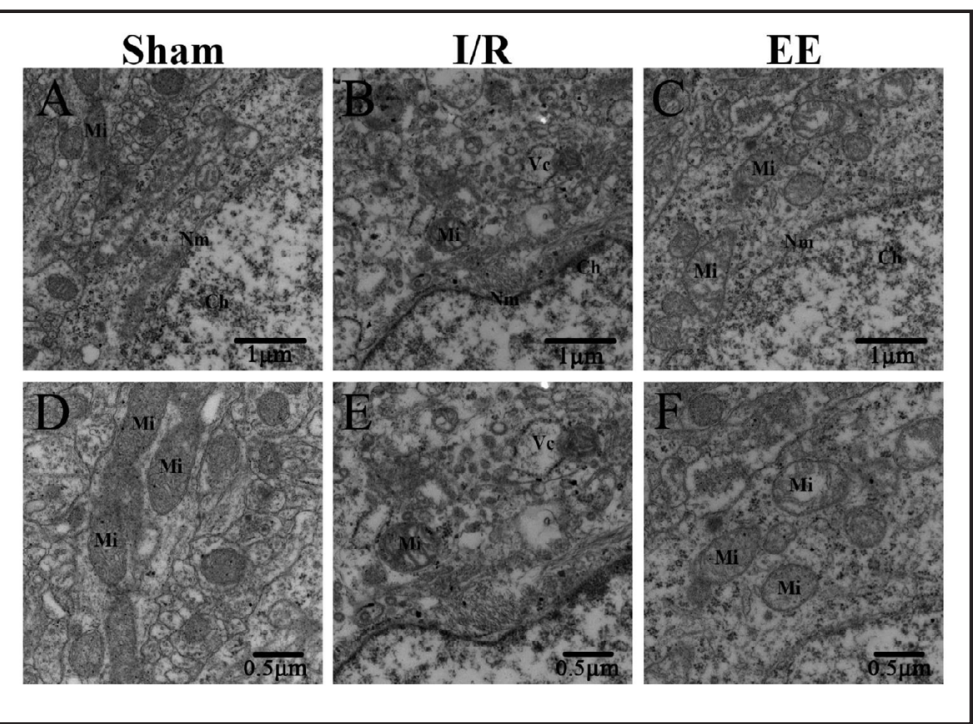

morphology and ultrastructure in the periinfarct cortex, transmission electron microscope was performed. Normal cortical neurons had large oval nucleus with clear nuclear membrane, even chromatin (Fig. 3A) and normal mitochondria (Fig. 3D). While in I/R group, neurons had irregular nuclear membrane, chromatin condensation, many vacuole (Fig. 3B) 


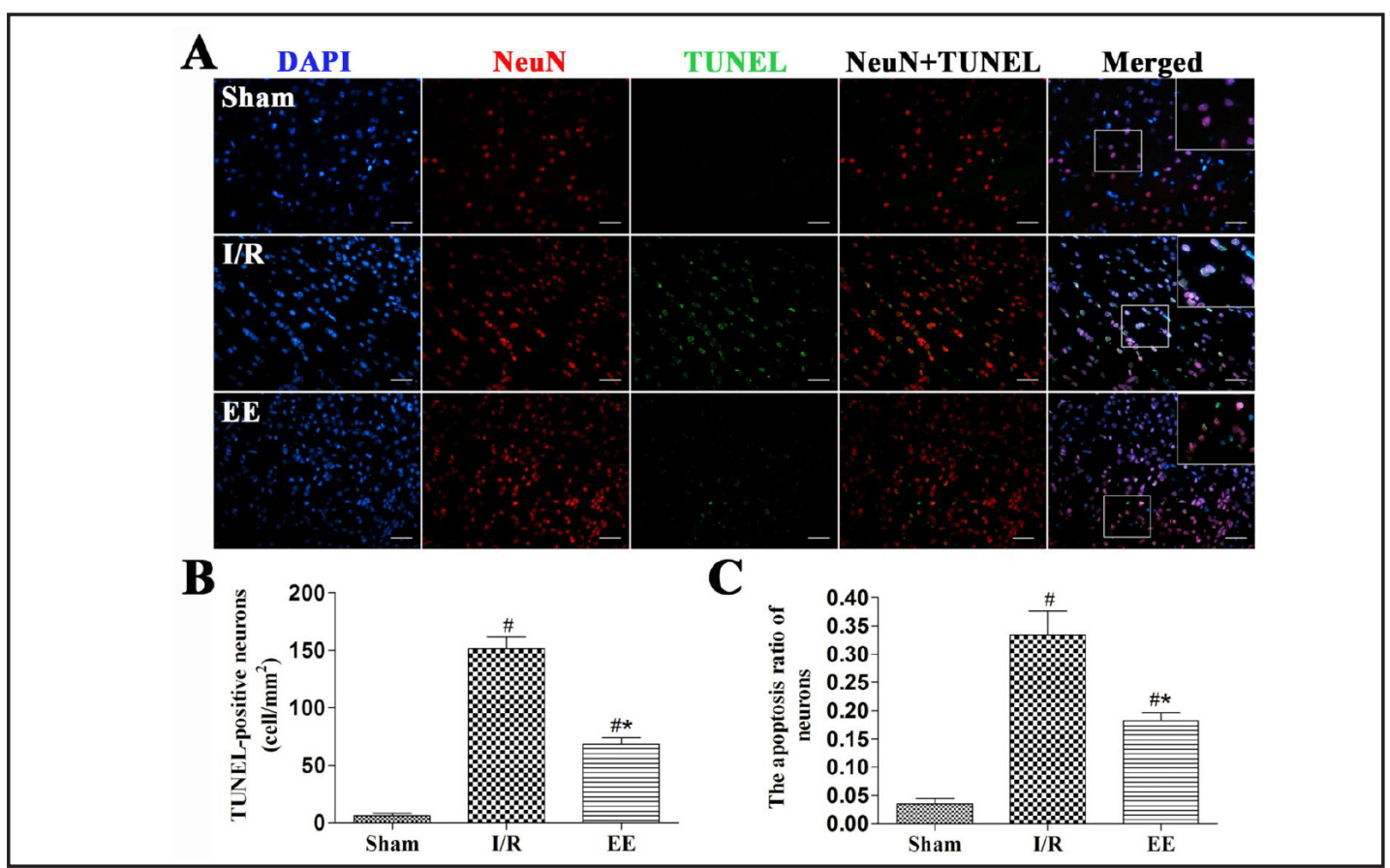

Fig. 4. EE treatment reduces neuronal apoptosis in the periinfarct cortex at 14 days after MCAO. (A) Double staining of TUNEL with NeuN for evaluation of the neuronal apoptosis in the periinfarct cortex, bar $=50 \mu \mathrm{m}$. Quantitative analysis for the number of TUNEL-positive neurons (B) and the apoptotic ratio of neurons (C) using Image Pro Plus 6.0. Data were presented as mean $\pm \mathrm{SD}, \mathrm{n}=6$ /group. ${ }^{*} P<0.05$ vs sham group, ${ }^{*} P<0.05$ vs I/R group.

and swollen mitochondria (Fig. 3E). By contrast, the above changes were alleviated in EE group (Fig. $3 \mathrm{C}$ and $3 \mathrm{~F}$ ). These results indicate that $\mathrm{EE}$ treatment alleviates neurons damage.

EE treatment decreases neuronal apoptosis in the periinfarct cortex

To directly clarify whether EE treatment reduced neuronal apoptosis in the periinfarct cortex and locate the apoptotic neurons, the double labeling of TUNEL with NeuN was conducted at 14 days after MCAO. A large number of TUNEL-positive neurons were observed in the periinfarct cortex of ischemic rats (I/R and EE), whereas TUNEL-positive neurons were seldom detected in sham group (Fig. 4A). EE treatment significantly decreased the number of TUNEL-positive neurons and the apoptosis ratio of neurons compared to I/R group (Fig. 4B and 4C). These findings supported the inhibitory effect of EE treatment on neuronal apoptosis.

EE treatment regulates the expressions of apoptosis-related proteins

To further investigate how EE treatment inhibited neuronal apoptosis in the periinfarct cortex, we tested the expression levels of antiapoptotic protein B cell lymphoma/leukemia-2 (Bcl-2), proapoptotic protein Bcl-2-associated X protein (Bax), cytochrome $c$ and caspase-3 in the periinfarct cortex at 14 days after MCAO. Western blot results showed that Bcl-2 protein levels were significantly increased following the EE treatment, while Bax, cytochrome $c$ and caspase-3 expressions were decreased (Fig. 5A-D). Moreover, the ratio of Bax/Bcl-2 in the EE group was obviously decreased when compared with I/R group (Fig. 5F).

Our immunohistological data suggested that Bcl-2, Bax and caspase-3 were mainly expressed in neurons (Fig. 6A), implicating that neuronal apoptosis accounts for the major portion of apoptosis. Rats in EE group showed higher expression levels of Bcl- 2 and lower expression levels of Bax and caspase- 3 than those in I/R group (Fig. 6B). These results suggest that EE treatment inhibits neuronal apoptosis via regulating apoptosis-related proteins expression in the periinfarct cortex following cerebral I/R injury. 
Fig. 5. EE treatment regulates the expressions of apoptosis-related proteins in the periinfarct cortex at 14 days after MCAO. (a) Western blots and quantitative analysis for Bcl-2 (A), Bax (B), cytochrome $c(\mathrm{C})$ and caspase-3 (D). (E) EE treatment downregulated the ratio of Bax/Bcl-2 compared with the I/R group. Data were presented as mean $\pm \mathrm{SD}, \mathrm{n}=6$ / group. ${ }^{\#} P<0.05$ vs sham group, ${ }^{*} P<0.05$ vs I/Rgroup.

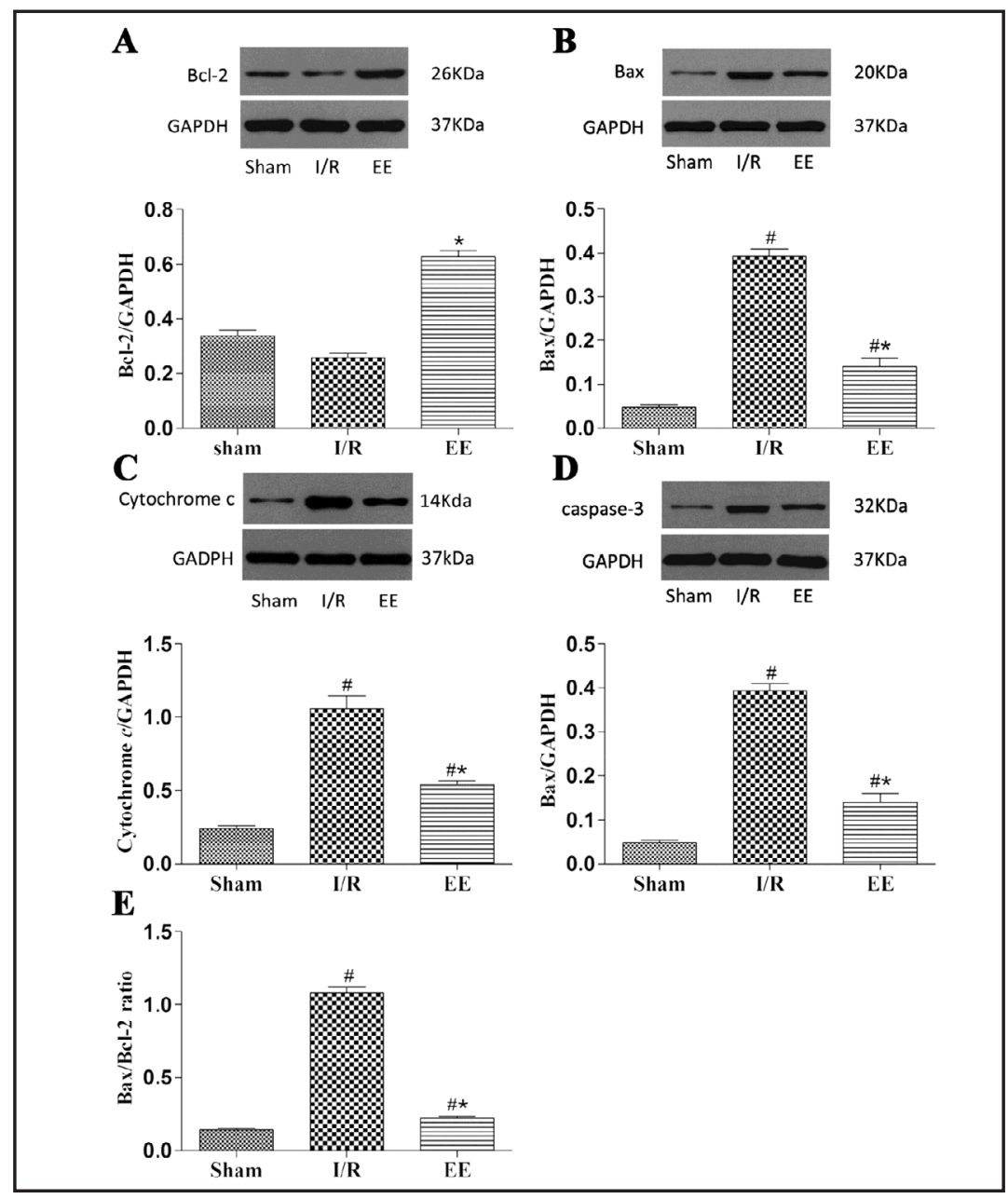

Fig. 6. Bcl-2, Bax and caspase-3 were measured by immunohistochemistry and immunofluorescence in the periinfarct cortex at 14 days after MCAO. (A) Representative photographs of immunohistochemical staining of Bcl-2 and Bax and immunofluorescent staining of caspase-3, bar $=50 \mu \mathrm{m}$. (B) Quantification analysis for the expression levels of Bcl-2, Bax and caspase-3 through measuring the integral optical density (IOD). Data were presented as mean $\pm \mathrm{SD}, \mathrm{n}=6$ /group. ${ }^{*} P<0.05$ vs sham group, ${ }^{*} P<0.05$ vs I/R group.

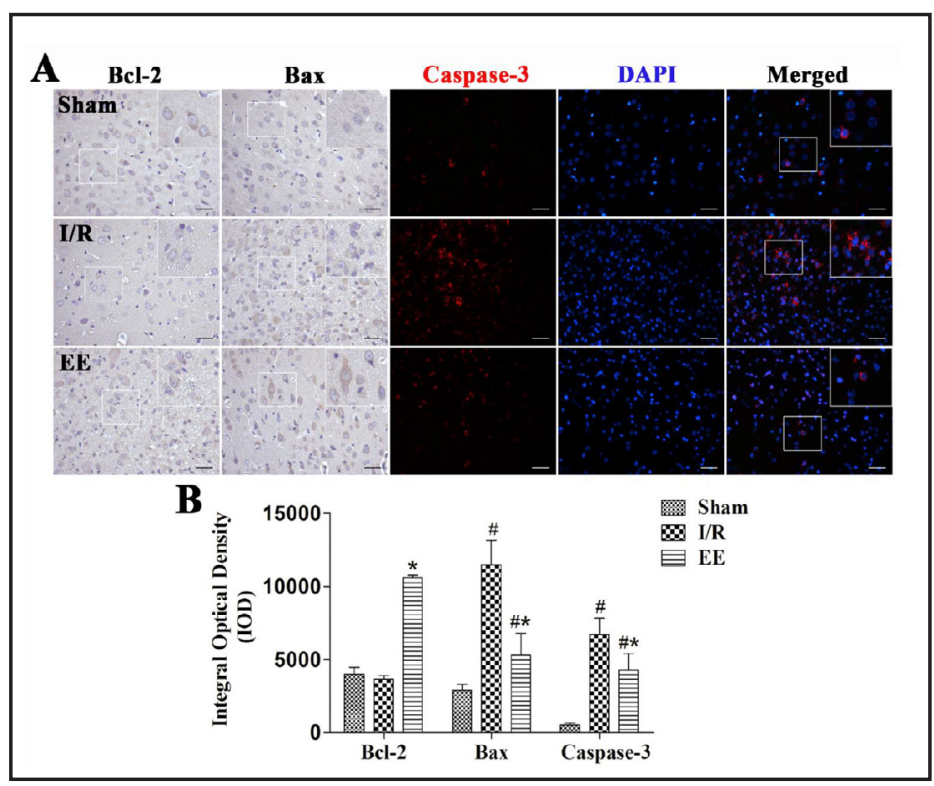

\section{Discussion}

Although EE treatment has been shown to be effective in reducing cerebral I/R-induced brain injury, the underlying mechanism is still hazy. To our knowledge, the current study 
was the first to investigate the effect of EE treatment on neuronal apoptosis in the periinfarct cortex in a MCAO rat model. Our data showed that EE treatment reduced neuronal apoptosis in the periinfarct cortex, increased Bcl-2 protein levels while decreased Bax, cytochrome $c$ and caspase-3 expressions following cerebral I/R injury. Furthermore, EE treatment improved neurological function of stroke animals, reduced infarct volume, increased the number of viable neurons and alleviated morphological and ultrastructural damage of neurons. Our findings suggest that reduction of neuronal apoptosis in the periinfarct cortex induced by EE treatment may be one of the possible mechanisms underlying its neuroprotective effect.

While most of the studies which tried to elucidate the possible mechanisms underlying the neuroprotective effect of EE treatment have focused on neurogenesis [23], synaptogenesis $[24,25]$ or angiogenesis [12], the importance of neuron survival has been neglected. As apoptosis is one of the major causes that lead to cell death after I/R injury especially in the periinfarct cortex [26], suppressing apoptosis may be an effective way to improve the survival rate of neurons and eventually benefit the prognosis after stroke. Previous studies have demonstrated that cellular apoptosis can be inhibited by medicines or stem cell transplantation in animal models of cerebral ischemia [27-29]. This study for the first time evaluated EE treatment-induced inhibition of neuronal apoptosis in the periinfarct cortex after cerebral I/R injury, which offered a new insight into the underlying mechanism of EE's neuroprotective effect.

Two general pathways including the extrinsic and intrinsic pathways are involved in the apoptotic process. The intrinsic pathway is closely related with the mitochondria which plays an important role in cell events including energy metabolism, cell differentiation and generation of reactive oxygen species (ROS) [5, 30]. Cerebral ischemia disrupts the normal state of electrochemical gradient in mitochondrial membrane, which is essential for normal function of mitochondria [31]. Damaged mitochondria could release cytochrome $c$ to the cytoplasm, leading to the apoptosis [32]. Therefore, any strategy that can maintain the structural integrity of mitochondria, promote mitochondrial biogenesis and prevent mitochondrial dysfunction may have the potentiality to inhibit apoptosis. Previous study has shown that exercise could induce mitochondrial biogenesis by increasing the expression of mitochondrial biogenesis factors and mitochondrial protein after experimental stroke [33]. Further study indicated that exercise might improve mitochondrial function in the ischemic brain by preventing mitochondrial fission and promoting mitochondrial fusion [34]. In our study, results from transmission electron microscope showed that treatment with EE significantly alleviated the damage of mitochondria, suggesting that EE treatment may provide neuroprotective effect through maintaining the structural integrity of mitochondria.

Our data showed that EE treatment reduced neuronal apoptosis in the periinfarct cortex caused by cerebral I/R injury, which could be mediated by the mitochondrialdependent pathway. Bcl-2 family proteins are pivotal moderators of apoptosis, which play an important role in maintaining mitochondrial stabilization [35]. The binding of Bcl-2 and Bax could prevent the pore formation on the outer membranes of mitochondria, reduce the release of cytochrome $c$, inhibit the formation of the apoptosome and thus inhibit activation of caspase family [36]. Previous studies have shown that both passive and pre-ischemic exercise suppressed the apoptosis and reduced neurons loss in the periinfarct area via upregulating expression of Bcl-2 and maintaining the balance ratio of Bax/Bcl-2 [37-39]. Except for Bcl-2 family, caspase-3 is one of the most important downstream executors in the mitochondrial apoptotic pathway, which is considered as an indicator of neuronal apoptosis [40]. Previous studies have demonstrated that deletion and inhibition of caspase-3 provided neuroprotection through mediating neuronal death and rendering neurons resistant to ischemic injury in vivo and in vitro [41]. In the present study, we showed that EE treatment increased Bcl-2 protein levels, decreased Bax expression and Bax/Bcl-2 ratio. Furthermore, EE treatment inhibited the release of cytochrome $c$ from the mitochondria to cytoplasm and thus prevented the activation of caspase-3. Although the mechanism of EE treatmentinduced inhibition of neuronal apoptosis needs to be further investigated, our present study suggests that the mitochondrial pathway may be an important target underlying this.

\section{KARGER}




\section{Cellular Physiology Cell Physiol Biochem 2017;41:1445-1456

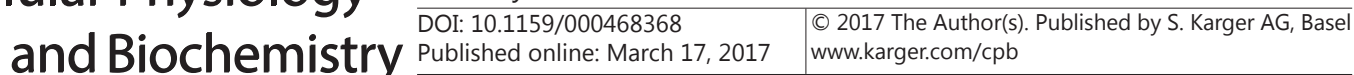 \\ Chen et al.: Enriched Environment Reduces Neuronal Apoptosis in Rats}

Taken together, the present study has demonstrated that EE treatment reduced neuronal apoptosis in the periinfarct area, which might contribute to the neuroprotective effect of EE against cerebral I/R injury. However, EE induced inhibition of apoptosis is a complex process, which claims for further research to explore the underlying mechanisms.

\section{Acknowledgements}

This work was financially supported by the National Natural Science Foundation of China (no.81173315).

\section{Disclosure Statement}

The authors declare that there is no conflict of interest.

\section{References}

1 Kernan WN, Ovbiagele B, Black HR, Bravata DM, Chimowitz MI, Ezekowitz MD, Fang MC, Fisher M, Furie KL, Heck DV, Johnston SC, Kasner SE, Kittner SJ, Mitchell PH, Rich MW, Richardson D, Schwamm LH, Wilson JA, American Heart Association Stroke Council, Council on Cardiovascular and Stroke Nursing, Council on Clinical Cardiology, Council on Peripheral Vascular Disease: Guidelines for the prevention of stroke in patients with stroke and transient ischemic attack: a guideline for healthcare professionals from the American Heart Association/American Stroke Association. Stroke 2014;45:2160-2236.

-2 Lackland DT, Roccella EJ, Deutsch AF, Fornage M, George MG, Howard G, Kissela BM, Kittner SJ, Lichtman JH, Lisabeth LD, Schwamm LH, Smith EE, Towfighi A, American Heart Association Stroke Council, Council on Cardiovascular and Stroke Nursing, Council on Quality of Care and Outcomes Research, Council on Functional Genomics and Translational Biology: Factors influencing the decline in stroke mortality: a statement from the American Heart Association/American Stroke Association. Stroke 2014;45:315-353.

-3 Bissonnette RP, Echeverri F, Mahboubi A, Green DR: Apoptotic cell death induced by c-myc is inhibited by bcl-2. Nature 1992;359:552-554.

4 Cho YS, Shin MS, Ko IG, Kim SE, Kim CJ, Sung YH, Yoon HS, Lee BJ: Ulinastatin inhibits cerebral ischemiainduced apoptosis in the hippocampus of gerbils. Mol Med Rep 2015;12:1796-1802.

5 Broughton BR, Reutens DC, Sobey CG: Apoptotic mechanisms after cerebral ischemia. Stroke 2009;40:331339.

6 Kuwana T, Mackey MR, Perkins G, Ellisman MH, Latterich M, Schneiter R, Green DR, Newmeyer DD: Bid, Bax, and lipids cooperate to form supramolecular openings in the outer mitochondrial membrane. Cell 2002;111:331-342.

7 Mitsios N, Gaffney J, Krupinski J, Mathias R, Wang Q, Hayward S, Rubio F, Kumar P, Kumar S, Slevin M: Expression of signaling molecules associated with apoptosis in human ischemic stroke tissue. Cell Biochem Biophys 2007;47:73-86.

-8 Mering S, Jolkkonen J: Proper housing conditions in experimental Stroke studies-special emphasis on environmental enrichment. Front Neurosci 2015;9:106.

-9 Briones TL, Rogozinska M, Woods J: Modulation of ischemia-induced NMDAR1 activation by environmental enrichment decreases oxidative damage. J Neurotrauma 2011;28:2485- 2492.

10 Li YW, Li QY, Wang JH, Xu XL: Contribution of p38 MAPK to the ameliorating effect of enriched environment on the cognitive deficits induced by chronic cerebral hypoperfusion. Cell Physiol Biochem 2016;40:549557.

11 White JH, Bartley E, Janssen H, Jordan LA, Spratt N: Exploring stroke survivor experience of participation in an enriched environment: a qualitative study. Disabil Rehabil 2015;37:593-600.

12 Zhang X, Chen XP, Lin JB, Xiong Y, Liao WJ, Wan Q: Effect of enriched environment on angiogenesis and neurological functions in rats with focal cerebral ischemia. Brain Res 2017;1655:176-185. 


\section{Cellular Physiology Cell Physiol Biochem 2017;41:1445-1456

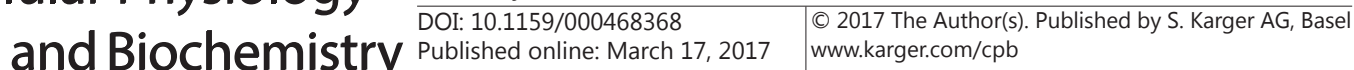

13 Fu CY, He XY, Li XF, Zhang X, Huang ZW, Li J, Chen M, Duan CZ: Nefiracetam attenuates pro-inflammatory cytokines and GABA transporter in specific brain regions of rats with post-ischemic seizures. Cell Physiol Biochem 2015;37:2023-2031.

14 Yu K, Wu Y, Hu Y, Zhang Q, Xie H, Liu G, Chen Y, Guo Z, Jia J: Neuroprotective effects of prior exposure to enriched environment on cerebral ischemia/reperfusion injury in rats: the possible molecular mechanism. Brain Res 2013;1538:93-103.

15 Chen J, Sanberg PR, Li Y, Wang L, Lu M, Willing AE, Sanchez-Ramos J, Chopp M: Intravenous administration of human umbilical cord blood reduces behavioral deficits after stroke in rats. Stroke 2001;32:2682-2688.

-16 Talley Watts L, Long JA, Boggs RC, Manga H, Huang S, Shen Q, Duong TQ: Delayed methylene blue improves lesion volume, multi-parametric quantitative magnetic resonance imaging measurements, and behavioral outcome after traumatic brain injury. J Neurotrauma 2016;33:194-202.

17 Zvejniece L, Svalbe B, Liepinsh E, Pulks E, Dambrova M: The sensorimotor and cognitive deficits in rats following 90- and 120-min transient occlusion of the middle cerebral artery. J Neurosci Methods 2012;208:197-204.

18 Han J, Pollak J, Yang T, Siddiqui MR, Doyle KP, Taravosh-Lahn K, Cekanaviciute E, Han A, Goodman JZ, Jones B, Jing D, Massa SM, Longo FM, Buckwalter MS: Delayed administration of a small molecule tropomyosinrelated kinase B ligand promotes recovery after hypoxic-ischemic stroke. Stroke 2012;43:1918-1924.

19 Huang J, Li Y, Tang Y, Tang G, Yang GY, Wang Y: CXCR4 antagonist AMD3100 protects blood-brain barrier integrity and reduces inflammatory response after focal ischemia in mice. Stroke 2013;44:190-197.

20 Wang H, Imamura Y, Ishibashi R, Chandana EP, Yamamoto M, Noda M: The Reck tumor suppressor protein alleviates tissue damage and promotes functional recovery after transient cerebral ischemia in mice. J Neurochem 2010;115:385-398.

21 Kwak HB, Song W, Lawler JM: Exercise training attenuates age-induced elevation in bax/bcl-2 ratio, apoptosis, and remodeling in the rat heart. FASEB J 2006;20:791-793.

-22 Lakshmi D, Gopinath K, Jayanthy G, Anjum S, Prakash D, Sudhandiran G: Ameliorating effect of fish oil on acrylamide induced oxidative stress and neuronal apoptosis in cerebral cortex. Neurochem Res 2012;37:1859-1867.

23 Monteiro BM, Moreira FA, Massensini AR, Moraes MF, Pereira GS: Enriched environment increases neurogenesis and improves social memory persistence in socially isolated adult mice. Hippocampus 2014;24:239-248.

24 Leggio MG, Mandolesi L, Federico F, Spirito F, Ricci B, Gelfo F, Petrosini L: Environmental enrichment promotes improved spatial abilities and enhanced dendritic growth in the rat. Behav Brain Res 2005;163:78-90.

25 He S, Ma J, Liu N, Yu X: Early enriched environment promotes neonatal GABAergic neurotransmission and accelerates synapse maturation. J Neurosci 2010;30:7910-7916.

26 Yuan J: Neuroprotective strategies targeting apoptotic and necrotic cell death for stroke. Apoptosis 2009;14:469-477.

-27 Tan Z, Turner RC, Leon RL, Li X, Hongpaisan J, Zheng W, Logsdon AF, Naser ZJ, Alkon DL, Rosen CL, Huber JD: Bryostatin improves survival and reduces ischemic brain injury in aged rats after acute ischemic stroke. Stroke 2013;44:3490-3497.

-28 Yu Q, Lu Z, Tao L, Yang L, Guo Y, Yang Y, Sun X, Ding Q: ROS-dependent neuroprotective effects of NaHS in ischemia brain injury involves the PARP/AIF pathway. Cell Physiol Biochem 2015;36:1539-1551.

-29 Bao C, Wang Y, Min H, Zhang M, Du X, Han R, Liu X: Combination of ginsenoside Rg1 and bone marrow mesenchymal stem cell transplantation in the treatment of cerebral ischemia reperfusion injury in rats. Cell Physiol Biochem 2015;37:901-910.

-30 Wang R, Liu YY, Liu XY, Jia SW, Zhao J, Cui D, Wang L: Resveratrol protects neurons and the myocardium by reducing oxidative stress and ameliorating mitochondria damage in a cerebral ischemia rat model. Cell Physiol Biochem 2014;34:854-864.

- 31 Chen SD, Yang DI, Lin TK, Shaw FZ, Liou CW, Chuang YC: Roles of oxidative stress, apoptosis, PGC-1 $\alpha$ and mitochondrial biogenesis in cerebral ischemia. Int J Mol Sci 2011;12:7199-7215.

-32 Sims NR, Muyderman H: Mitochondria, oxidative metabolism and cell death in stroke. Biochim Biophys Acta 2010;1802:80-91.

-33 Zhang Q, Wu Y, Zhang P, Sha H, Jia J, Hu Y, Zhu J: Exercise induces mitochondrial biogenesis after brain ischemia in rats. Neuroscience 2012;205:10-17. 


\section{Cellular Physiology Cell Physiol Biochem 2017;41:1445-1456 \begin{tabular}{ll|l} 
DOI: 10.1159/000468368 & O 2017 The Author(s). Published by S. Karger AG, Basel \\
www.karger.com/cpb
\end{tabular} \\ Published onlıne: IVarch 17, 2017 www.karger.com/cpb}

-34 Zhang L, He Z, Zhang Q, Wu Y, Yang X, Niu W, Hu Y, Jia J: Exercise pretreatment promotes mitochondrial dynamic protein OPA1 expression after cerebral ischemia in rats. Int J Mol Sci 2014;15:4453-4463.

35 Hu XL, Olsson T, Johansson IM: Dynamic changes of the anti- and pro-apoptotic proteins Bcl-w, Bcl-2, and Bax with Smac/Diablo mitochondrial release after photothrombotic ring stroke in rats. Eur J Neurosci 2004;20:1177-1188.

-36 Hengartner MO: The biochemistry of apoptosis. Nature 2000;407:770-776.

37 Seo TB, Kim TW, Shin MS, Ji ES, Cho HS, Lee JM, Kim TW, Kim CJ: Aerobic exercise alleviates ischemiainduced memory impairment by enhancing cell proliferation and suppressing neuronal apoptosis in hippocampus. Int Neurourol J 2014;18 :187-197.

38 Li M, Peng J, Wang MD, Song YL, Mei YW, Fang Y: Passive movement improves the learning and memory function of rats with cerebral infarction by inhibiting neuron cell apoptosis. Mol Neurobio 2014;49:216221.

39 Aboutaleb N, Shamsaei N, Khaksari M, Erfani S, Rajabi H, Nikbakht F: Pre-ischemic exercise reduces apoptosis in hippocampal CA3 cells after cerebral ischemia by modulation of the Bax/Bcl-2 proteins ratio and prevention of caspase-3 activation. J Physiol Sci 2015;65:435-443.

40 Xing J, Lu J: HIF- $1 \alpha$ activation attenuates IL-6 and TNF- $\alpha$ pathways in hippocampus of rats following transient global ischemia. Cell Physiol Biochem 2016;39:511-520.

- 41 Le DA, Wu Y, Huang Z, Matsushita K, Plesnila N, Augustinack JC, Hyman BT, Yuan J, Kuida K, Flavell RA, Moskowitz MA: Caspase activation and neuroprotection in caspase-3- deficient mice after in vivo cerebral ischemia and in vitro oxygen glucose deprivation. Proc Natl Acad Sci USA 2002;99:15188-15193. 